\title{
The Probabilistic Complexity of the Voronoi Diagram of Points on a Polyhedron
}

\author{
Mordecai J. Golin \\ Dept. of Computer Science \\ Hong Kong UST \\ golin@cs.ust.hk
}

\author{
Hyeon-Suk $\mathrm{Na}$ \\ Inria-Lorraine \\ na@loria.fr
}

\begin{abstract}
It is well known that the complexity, i.e., the number of vertices, edges and faces, of the 3-dimensional Voronoi diagram of $n$ points can be as bad as $\Theta\left(n^{2}\right)$. Interest has recently arisen as to what happens, both in deterministic and probabilistic situations, when the 3-dimensional points are restricted to lie on the surface of a 2-dimensional object. In this paper we consider the situation when the points are drawn from a 2-dimensional Poisson distribution with rate $n$ over a fixed union of triangles in $\mathbb{R}^{3}$. We show that with high probability the complexity of their Voronoi diagram is $\tilde{O}(n)$.
\end{abstract}

This implies, for example, that the complexity of the Voronoi diagram of points chosen from the surface of a general fixed polyhedron in $\mathbb{R}^{3}$ will also be $\tilde{O}(n)$ with high probability.

\section{Categories and Subject Descriptors}

G.3 [Mathematics of Computing]: Probability And Statistics; I.3.5 [Computing Methodologies]: Computer Graphics-Computational Geometry and Object Modeling

\section{General Terms}

Algorithms

\section{INTRODUCTION}

Let $P$ be a set of 3 -dimensional points. The Voronoi $\mathrm{Di}$ agram of the points and its dual, the Delaunay triangulation, are extremely well studied structures. The complexity, $|V D(P)|$ of the Voronoi diagram is the number of lower dimensional pieces of which it is composed, i.e., the total number of vertices, edges and faces and regions that it contains. It is well known that, in the worst case, the complexity can be as high as $\Theta\left(n^{2}\right)$ [6]. It has also been observed that, if the points are sampled from some types of restricted point sets, the complexity tends, in practice, to be much lower.

The problem of understanding the structure of the 3-dimensional

Permission to make digital or hard copies of all or part of this work for personal or classroom use is granted without fee provided that copies are not made or distributed for profit or commercial advantage and that copies bear this notice and the full citation on the first page. To copy otherwise, to republish, to post on servers or to redistribute to lists, requires prior specific permission and/or a fee.

SoCG'02, June 5-7, 2002, Barcelona, Spain

Copyright 2002 ACM 1-58113-504-1/02/0006 ..\$5.00
Voronoi diagram of point sets from 2-dimensional surfaces has begun to be of interest in recent years. This is because, as described in [1] and [3], Voronoi diagrams and Delaunay triangulations are of use in several geometric problems, e.g., surface reconstruction, mesh generation and surface modeling. In these problems a 2-dimensional surface is often sampled and then modeled, at least initially, by the Delaunay triangulation of the sample. Many parameters of such algorithms such as their running times and the complexity of their representations, then depend upon the complexity of the Delaunay triangulation (which is the same as that of the Voronoi diagram).

The two results [1] and [3] mentioned above seem to be the first to try and formally analyze the complexity of such Voronoi diagrams. In [1] Attali and Boissonnat prove that if $n$ "well-sampled" points are chosen from a polyhedral surface then the complexity of their Voronoi diagram is $O\left(n^{7 / 4}\right)$ where "well-sampled" is defined using the concept of local feature size; if the points are drawn in the same way from the surface of a convex polytope this reduces down to $O\left(n^{3 / 2}\right) .{ }^{1}$ In [3] Erikson proves that there is a set of $n$ "well-sampled" points from the cylinder with Voronoi diagram complexity $\Omega\left(n^{3 / 2}\right)$.

Working from a probabilistic perspective the authors of this work showed [4] that if points are drawn from a 2-dimensional Poisson distribution with rate $n$ from the surface of a fixed convex polytope then the expected complexity of the Voronoi diagram of the points would be $O(n)$ (with the same result also holding if $n$ points were chosen IID uniformly from the surface of the polytope).

The major result of this paper is to prove a high probability theorem when points are drawn from the surface of a collection of triangles. More specifically

TheOREM 1. Let $\mathcal{F}=\left\{F_{1}, \ldots, F_{k}\right\}$ be a collection of $k$ triangles in $\mathbb{R}^{3}$. Let $P_{n}$ be a set of points drawn from a 2dimensional Poisson distribution on $\mathcal{F}$ with rate $n$. Then $\operatorname{Pr}\left(\left|V D\left(P_{n}\right)\right|=\tilde{O}(n)\right)=1-n^{-\Omega(\log n)}$.

\footnotetext{
${ }^{1}$ Just prior to submission we learnt of new work [2] by Attali and Boissonnat that proves a linear bound on the complexity of the Delaunay Triangulation of $n$ points well-sampled from a polyhedral surface (using a slightly revised definition of well-sampled). This seems to be the deterministic analogue of the result in this current paper.
} 
Note that since a polyhedron can be decomposed into a finite set of triangles this immediately implies that if $P_{n}$ is a set of points drawn from the standard 2-dimensional Poisson distribution with rate $n$ on the surface of a fixed polyhedron then $\operatorname{Pr}\left(\left|\operatorname{VD}\left(P_{n}\right)\right|=\tilde{O}(n)\right)=1-n^{-\Omega(\log n)}$.

We start by defining some of the terms used in the theorem. A triangle will denote a closed triangle that contains its interior and edges.

A Poisson distribution on $\mathcal{F}$ with rate $n$ is the distribution in which, for every measurable $M \subset \cup_{i} F_{i}$, we have that $N(M)$, the number of points in $M$, satisfies $\operatorname{Pr}(N(M)=$ $k)=\frac{(n \operatorname{Area}(M))^{k} e^{-(n \operatorname{Area}(M))}}{k !}$ and for $M, M^{\prime} \subset F$ with $M \cap M^{\prime}=\emptyset, N(\stackrel{k !}{M})$ and $N\left(M^{\prime}\right)$ are independent. The expected number of points in $P_{n}$ will be $\operatorname{Area}(\mathcal{F}) \cdot n$.

$g(n)=\tilde{O}(f(n))$ denotes that $g(n)=O\left(f(n) \log ^{i} n\right)$ for some fixed $i$., i.e., it "hides" $\log n$ factors. In Theorem 1 as proved in this paper the $\tilde{O}(n)$ is actually a $O\left(n \log ^{6} n\right)$ term. The reason for using the tilde notation is that it vastly simplifies the proofs by allowing us to lump many cases together and makes them more readible.

Also, with slight modifications to the proofs, all of the probabilistic results given in this paper will hold if we change the distribution so that $P_{n}$, instead of being drawn from a Poisson distribution, is a set of $n$ points independently identically distributed uniformly from $\mathcal{F}$.

In the next section we discuss how to reduce the proof of Theorem 1 to a case-by-case analysis of a simpler problem. In Section 3 we introduce some tools that we will need in our proof and in Section 4 we perform the case-by-case analysis.

Before ending this section we review some notation that we will be using: For a point $p \in \mathbb{R}^{3}$ and any set $X \subseteq \mathbb{R}^{3}$ extend the Euclidean distance function so that $d(p, X)=$ $\inf _{q \in X} d(p, q)$. Also set $N N(p, X)$ to be a nearest neighbor to $p$ in $X$, i.e., a $q \in X$ such that $d(p, q)=d(p, X)$. In this paper $X$ will always be a closed polygonal piece so $N N(p, X)$ will be unique.

For $r>0$ define

$$
S(p, r)=\left\{q \in \mathbb{R}^{3}: d(q, p) \leq r\right\}
$$

to be the closed ball of radius $r$ around $p$.

Let $\Pi$ be a plane and $p \in \Pi$. Then define $C_{\Pi}(p, r)=\{q \in$ $\Pi: d(q, p) \leq r\}$ to be the closed disc of radius $r$ on $\Pi$ centered at $p$.

Finally, for $F_{1}, F_{2}$ triangles and $\Pi_{1}, \Pi_{2}$ their supporting planes, we say that $F_{1} \| F_{2}$ if $\Pi_{1}$ is parallel to $\Pi_{2}$ and $F_{1} \nvdash F_{2}$ otherwise.

Important Note: In this extended abstract we only provide the upper level description of some of the proofs and omit some details.

\section{REDUCTIONS}

Instead of calculating the complexity $\left|V D\left(P_{n}\right)\right|$ directly we will instead bound the number of Voronoi spheres corresponding to Voronoi faces:

A Voronoi sphere is a sphere that has at least one point of $P_{n}$ on its boundary and no other points of $P_{n}$ in its interior. We count the number of combinatorially different spheres where spheres are considered to be different only if they have different set of points of $P_{n}$ on the boundary. Also, since the event of points in a $P_{n}$ chosen from the Poisson distribution being in general position has probability 1 , we can assume that each Voronoi sphere has at most 4 points on its surface. Thus every vertex/edge/face/region of $\operatorname{VD}\left(P_{n}\right)$ corresponds to Voronoi sphere $S=S(p, r)$ with $4 / 3 / 2 / 1$ points of $P_{n}$ on its boundary, i.e., the complexity of $\operatorname{VD}\left(P_{n}\right)$ is bounded by the number of possible Voronoi spheres having different $4 / 3 / 2 / 1$ tuple of points of $P_{n}$ on its boundary.

Noting that every edge corresponds to a triple of points chosen from the 4 points corresponding to some Voronoi vertex and every face corresponds to a pair of points chosen from the 4 points corresponding to some Voronoi vertex, we see the complexity of $\operatorname{VD}\left(P_{n}\right)$ is proportional to the number of Voronoi vertices. It therefore suffices to count the number of Voronoi vertices.

Furthermore, as recently pointed out by Attali and Boissonnat [2], Euler's relations imply that the number of tetrahedra in the 3-D Delaunay triangulation of $n$ sites is linear in the number of edges in this triangulation; by taking the dual we have that the number of Voronoi vertices in the 3-D Voronoi diagram is actually linear in the number of Voronoi faces. So, the size of $\operatorname{VD}\left(P_{n}\right)$ is bounded by the number of Voronoi spheres defining Voronoi faces, i.e., the Voronoi spheres defined by exactly two points. To simplify matters, in the rest of this paper we will therefore assume that $V D\left(P_{n}\right)$ is not the full set of Voronoi spheres but only those corresponding to Voronoi faces, i.e., those defined by two points in $P_{n}$.

To further simplify matters we will also assume that the pair $p, p^{\prime} \in P_{n}$ defining the Voronoi spheres are not both on the same triangle. The justification for this assumption is that if $p, p^{\prime}$ were on the same triangle $F_{i}$ then the intersection of their empty Voronoi sphere with $F_{i}$ would be an empty circle in $F_{i}$. This implies that $p, p^{\prime}$ are Voronoi neighbors, i.e., define an edge, in the two-dimensional Voronoi diagram of $P_{n} \cap F_{i}$ on $\Pi_{i}$, the supporting plane of $F_{i}$. The two dimensional Voronoi diagram is linear in the number of sites so the number of such faces is $O\left(\left|P_{n} \cap F_{i}\right|\right)$. Summing over all $F_{i}$ we have that the total number of Voronoi spheres defined by $p, p^{\prime}$ with both points on the same triangle is $\sum_{i} O\left(\left|P_{n} \cap F_{i}\right|\right)=O\left(\left|P_{n}\right|\right)$. Let $A=\operatorname{Area}(\mathcal{F})=$ $\sum_{i} \operatorname{Area}\left(F_{i}\right)$. From the Poisson distribution it is easy to work out that $\operatorname{Pr}\left(\left|P_{n}\right| \geq 2 A n\right)=n^{-\Omega(\log n)}$ so, with probability $1-n^{-\Omega(\log n)}$, the number of such faces is $O(n)$. Thus, in the sequel, we will assume that $\operatorname{VD}\left(P_{n}\right)$ is the set of Voronoi spheres defined by two points in $P_{n}$ such that the two points do not lie on the same triangle $F_{i}$.

Bounding the probabilistic complexity of $\operatorname{VD}\left(P_{n}\right)$ directly can be quite difficult since the conditionality of assuming that certain spheres are Voronoi skews the rest of the distri- 
bution. In this section we show how to reduce the problem to a more manageable one. This will require introducing new definitions and utility lemmas. In everything that follows it is implicitly assumed that $n$ is fixed.

Definition 1. Let $F \subset \mathbb{R}^{3}$ be a triangle, $p \in \mathbb{R}^{3}$ and $r \geq 0$. Then $S(p, r)$ is an $F$-good sphere if $\operatorname{Area}(S(p, r) \cap$ $F) \leq \frac{\log ^{2} n}{n}$.

If $\mathcal{F}=\left\{F_{1}, \ldots, F_{k}\right\}$ is a collection of triangles then $S(p, r)$ is an $\mathcal{F}$-good sphere if $S(p, r)$ is an $F_{i}$-good sphere for every triangle $F_{i}$.

The important observation is

LEMma 1 . Let $\mathcal{F}=\left\{F_{1}, \ldots, F_{k}\right\}$ be a collection of $k$ triangles in $\mathbb{R}^{3}$. Let $P_{n}$ be a set of points drawn from a 2dimensional Poisson distribution on $\mathcal{F}$ with rate $n$. Then

$$
\begin{aligned}
& \operatorname{Pr}\left(\text { all Voronoi spheres in } \operatorname{VD}\left(P_{n}\right) \text { are } \mathcal{F} \text {-good }\right) \\
& =1-n^{-\Omega(\log n)} .
\end{aligned}
$$

The inituition behind this proof is that if a given sphere is not $\mathcal{F}$-good then the probability that it contains no points of the Poisson distributed $P_{n}$ is no more than $e^{-\frac{\log ^{2} n}{n} n}=$ $n^{-\Omega(\log n)}$. This intuition can be formalized into a rigorous proof (a similar lemma was proved in [4] for $\mathcal{F}$ the boundary of a convex polygon.

We now define something easier to bound than Voronoi spheres:

Definition 2. Let $\mathcal{F}=\left\{F_{1}, \ldots, F_{k}\right\}$ be a collection of triangles, $X \subseteq \mathbb{R}^{3}$ and $P \subseteq \cup_{i} F_{i}$. Now define

$$
\begin{aligned}
& D(P)=\left\{\begin{array}{ll}
\left\{p_{1}, p_{2}\right\}: & p_{1}, p_{2} \text { are not on the same face } F_{i} \\
& \text { and }\left\{p_{1}, p_{2}\right\} \subseteq P \cap S(p, r) \\
& \text { for some } \mathcal{F} \text {-good sphere } S(p, r)
\end{array}\right\} \\
& D_{X}(P)=\left\{\begin{array}{r}
p_{1}, p_{2} \text { are not on the same face } F_{i} \\
\left\{p_{1}, p_{2}\right\}: \text { and }\left\{p_{1}, p_{2}\right\} \subseteq P \cap S(p, r) \text { for some } \\
\mathcal{F} \text {-good sphere } S(p, r) \text { with } p \in X
\end{array}\right\}
\end{aligned}
$$

Note that if some Voronoi Sphere $S=S(p, r)$ in $\operatorname{VD}\left(P_{n}\right)$ containing 2 points $\left\{p_{1}, p_{2}\right\}$ on its boundary is a $\mathcal{F}$-good sphere then $\left\{p_{1}, p_{2}\right\} \in D\left(P_{n}\right)$ (recall that we defined $\operatorname{VD}\left(P_{n}\right)$ so that the two defining points can not lie on the same triangle). So, if all Voronoi spheres in $V D\left(P_{n}\right)$ are $\mathcal{F}$-good spheres then $\left|V D\left(P_{n}\right)\right| \leq\left|D\left(P_{n}\right)\right|$. Combining this with Lemma 1 gives that

$$
\begin{aligned}
& \operatorname{Pr}\left(\left|D\left(P_{n}\right)\right|=\tilde{O}(n)\right)=1-n^{-\Omega(\log n)} \\
& \text { implies } \operatorname{Pr}\left(\left|\operatorname{VD}\left(P_{n}\right)\right|=\tilde{O}(n)\right)=1-n^{-\Omega(\log n)} .
\end{aligned}
$$

We will now devote ourselves to proving
Theorem 2. Let $\mathcal{F}=\left\{F_{1}, \ldots, F_{k}\right\}$ be a collection of $k$ triangles in $\mathbb{R}^{3}$. Let $P_{n}$ be a set of points drawn from a 2dimensional Poisson distribution on $\mathcal{F}$ with rate $n$. Then

$$
\operatorname{Pr}\left(\left|D\left(P_{n}\right)\right|=\tilde{O}(n)\right)=1-n^{-\Omega(\log n)} .
$$

From the discussion above, proving Theorem 2 implies Theorem 1 .

We can actually go one step further and notice that, since (i) if $S(p, r)$ is a $\mathcal{F}$-good sphere with respect to $P_{n}$ then $S(p, r)$ is a $\left\{F_{i_{1}}, F_{i_{2}}\right\}$-good sphere for any $1 \leq i_{1}, i_{2} \leq k$ and (ii) $D\left(P_{n}\right)$ is defined by a pair of points, we have

$$
D\left(P_{n}\right) \subseteq \bigcup_{1 \leq i_{1}, i_{2} \leq k} D\left(P_{n} \cap\left(F_{i_{1}} \cup F_{i_{2}}\right)\right) .
$$

So

$$
\left|D\left(P_{n}\right)\right| \leq \sum_{1 \leq i_{1}, i_{2} \leq k}\left|D\left(P_{n} \cap\left(F_{i_{1}} \cup F_{i_{2}}\right)\right)\right|
$$

Suppose we had

THEOREM 3. Let $\mathcal{F}=\left\{F_{1}, F_{2}\right\}$ be a pair of triangles in $\mathbb{R}^{3}$. Let $P_{n}$ be a set of points drawn from a 2-dimensional Poisson distribution on $\mathcal{F}$ with rate $n$. Then

$$
\operatorname{Pr}\left(\left|D\left(P_{n}\right)\right|=\tilde{O}(n)\right)=1-n^{-\Omega(\log n)} .
$$

One of the properties of the Poisson distribution is that if $P_{n}$ is a set of points drawn from a 2-dimensional Poisson distribution on $\mathcal{F}$ with rate $n$ and $X \subset \mathcal{F}$ is measurable, then $P_{n} \cap X$ has the same distribution as a set of points drawn from a 2-dimensional Poisson distribution on $X$ with rate $n$. Therefore, combining Theorem 3 and (1) would imply Theorem 2. Thus, to prove Theorem 2 it suffices to prove Theorem 3 .

From now on we will therefore assume that $\mathcal{F}=\left\{F_{1}, F_{2}\right\}$ is composed of two triangles. Our approach to proving Theorem 3 will be to split $D\left(P_{n}\right)$ up into managable pieces. We do this as follows:

Definition 3. Let $F \subset \mathbb{R}^{3}$ be a triangle. Set $V(F), E(F)$ and $I(F)$, to be, respectively, the vertices, edges and interior of $F$. That is, $V(F)$ are the three vertices of $F, E(F)$ the three edges minus the vertices and $I(F)$, the triangle without its edges.

For any $p \in \mathbb{R}^{3}$ set

$$
r_{p}=\max \{r: S(p, r) \text { is a } \mathcal{F} \text {-good sphere }\} .
$$

For $i=1,2$, define $L_{i}(p) \in\{\emptyset, V, E, I\}$ such that

$L_{i}(p)= \begin{cases}\emptyset & \text { if } S\left(p, r_{p}\right) \cap F_{i}=\emptyset \\ V & \text { if } S\left(p, r_{p}\right) \cap F_{i} \neq \emptyset \text { and } N N\left(p, F_{i}\right) \in V\left(F_{i}\right) \\ E & \text { if } S\left(p, r_{p}\right) \cap F_{i} \neq \emptyset \text { and } N N\left(p, F_{i}\right) \in E\left(F_{i}\right) \\ I & \text { if } S\left(p, r_{p}\right) \cap F_{i} \neq \emptyset \text { and } N N\left(p, F_{i}\right) \in I\left(F_{i}\right)\end{cases}$ 


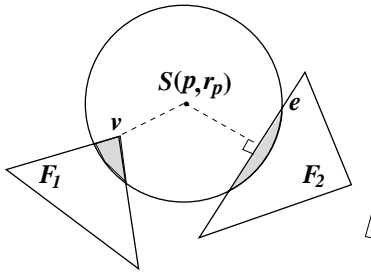

(a)

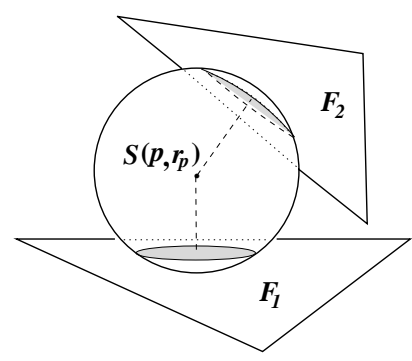

(c)

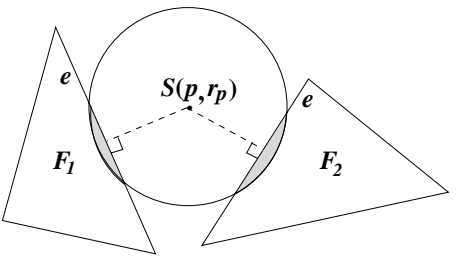

(b)

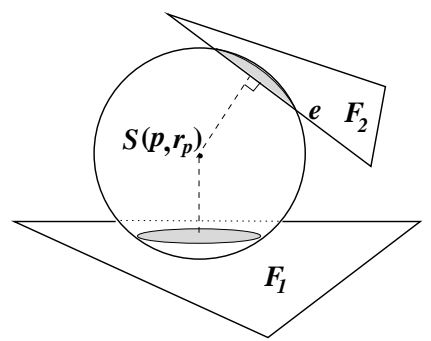

(d)
Figure 1: Different ways for spheres to intersect triangles: (a) $L=(V, E)$; (b) $L=(E, E)$; (c) $L=(I, I)$; (d) $L=(I, E)$. The cases $L=(V, I)$ and $L=(V, V)$ are not illustrated.

$L=\left(L_{1}, L_{2}\right)$ will be the label of $p$. Figure 1 illustrates ways in which a sphere can intersect two triangles and their labels. For any given label $L=\left(L_{1}, L_{2}\right) \in\{\emptyset, V, E, I\}^{2}$ we can define

$$
R_{L}=\left\{p \in \mathbb{R}^{3}: L(p)=L\right\} .
$$

Since every $p \in \mathbb{R}^{3}$ has a unique label $L(p)$ the $R_{L}$ form a partition of $\mathbb{R}^{3}$ into 15 regions (label $(\emptyset, \emptyset)$ can trivially not occur). Note that this partition depends upon $\mathcal{F}$ and $n$ but not on the Poisson distribution.

Now, let $P_{n}$ be any finite set of points in $\mathbb{R}^{3}$ (not necessarily a random one). Since every pair $\left\{p_{1}, p_{2}\right\} \in D\left(P_{n}\right)$ is contained in at least one $\mathcal{F}$-good sphere $S(p, r)$ that intersects both $F_{1}$ and $F_{2}$ and every $p$ is in exactly one $R_{L}$, we have

$$
D\left(P_{n}\right)=\bigcup_{L \in\{V, E, I\}^{2}} D_{R_{L}}\left(P_{n}\right)
$$

so $\left|D\left(P_{n}\right)\right| \leq \sum_{L \in\{V, E, I\}^{2}}\left|D_{R_{L}}\left(P_{n}\right)\right|$. Our major theorem is:

TheOREM 4. Let $\mathcal{F}=\left\{F_{1}, F_{2}\right\}$ be a pair of triangles in $\mathbb{R}^{3}$ and $P_{n}$ a set of points drawn from a 2-dimensional Poisson distribution on $\mathcal{F}$ with rate $n$. Let $L \in\{V, E, I\}^{2}$ be an arbitrary fixed label. Then

$$
\operatorname{Pr}\left(\left|D_{R_{L}}\left(P_{n}\right)\right|=\tilde{O}(n)\right)=1-n^{-\Omega(\log n)} .
$$

Since this is true for all labels $L$ and there are only 9 different labels, summing them gives

$$
\operatorname{Pr}\left(\left|D\left(P_{n}\right)\right|=\tilde{O}(n)\right)=1-n^{-\Omega(\log n)}
$$

proving Theorem 3 which, as discussed before, implies Theorem 1.

We have therefore just demonstrated that to prove Theorem 1 it suffices to prove Theorem 4 . The sequel of this paper will be devoted to proving this.

\section{TOOLS}

In this section we introduce the major tools that we will use in our proof. In what follows we always assume that $\mathcal{F}=\left\{F_{1}, F_{2}\right\}$ is a pair of triangles.

Definition 4. Let $F \subset \mathbb{R}^{3}$ be a triangle, $p^{\prime} \in F$ and $X \subseteq F$. Define

$$
\begin{aligned}
& \mathcal{G}_{F}\left(p^{\prime}\right)=\left\{S(p, r): \begin{array}{l}
N N(p, F)=p^{\prime} \text { and } \\
S(p, r) \text { is an F-good sphere }
\end{array}\right\} \\
& M_{F}\left(p^{\prime}\right)=\bigcup_{S(p, r) \in \mathcal{G}_{F}\left(p^{\prime}\right)}(S(p, r) \cap F) \\
& M_{F}(X)=\bigcup_{p^{\prime} \in X} M_{F}\left(p^{\prime}\right) .
\end{aligned}
$$

Intuitively $M_{F}\left(p^{\prime}\right)$ is the set of all points in $F$ that can belong to some $F$-good sphere such that if $p$ is the center of the sphere then $N N(p, F)=p^{\prime}$.

Definition 5. Let $p \in \mathbb{R}^{3}$ be fixed. For $i=1,2$, set

$$
G_{i}(p)= \begin{cases}M_{F_{i}}\left(N N\left(p, F_{i}\right)\right) & L_{i}(p) \neq \emptyset \\ \emptyset & L_{i}(p)=\emptyset\end{cases}
$$

and define $G(p)=\cup_{i=1}^{2} G_{i}(p)$.

For $X \subseteq \mathbb{R}^{3}$ set $G_{i}(X)=\cup_{p \in X} G_{i}(p)$ and $G(X)=\cup_{i=1}^{2} G_{i}(X)=$ $\cup_{p \in X} G(p)$.

The important thing to notice is that $G(p)$ contains all points that can be contained by some $F_{i}$-good sphere centered at $p$. More formally, if $S(p, r)$ is an $F_{i}$-good sphere then, by definition, $S(p, r) \cap F_{i} \subseteq G_{i}(p)$; thus, if $\left\{p_{1}, p_{2}\right\} \subset$ $S(p, r)$ for some $\mathcal{F}$-good sphere then $\left\{p_{1}, p_{2}\right\} \subset G(p)$. This in turn implies

Lemma 2. Let $P_{n} \subset \mathbb{R}^{3}$ be finite and $X \subseteq \mathbb{R}^{3}$. Then

$$
\left|D_{X}\left(P_{n}\right)\right| \leq\left|P_{n} \cap G(X)\right|^{2} \text {. }
$$

Noting the fact that if $X \subset \mathbb{R}^{3}$ is measurable in $\mathbb{R}^{3}$ then $G(X)$ is measurable in $\mathcal{F}$ and using the definition of the Poisson distribution we can then prove:

Corollary 3. Let $X \subset \mathbb{R}^{3}$ be measurable and $f>0$ such that Area $(G(X)) \leq f / n$. Let $P_{n}$ be a set of points drawn from a 2-dimensional Poisson distribution on $\mathcal{F}$ with rate $n$. Then

$$
\operatorname{Pr}\left(\left|D_{X}\left(P_{n}\right)\right| \geq(f \log n)^{2}\right)=n^{-\Omega(\log n)} .
$$




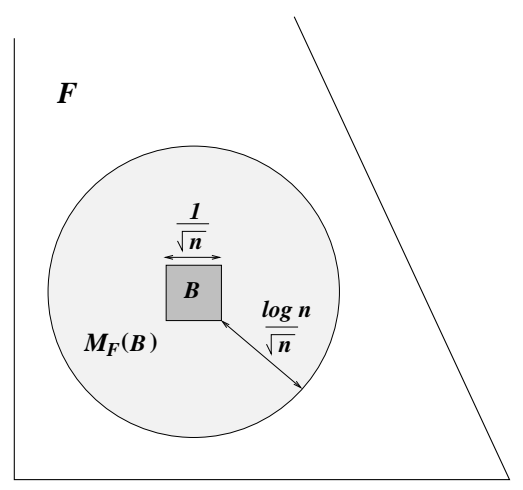

Figure 2: Illustration of Lemma 4 (1) and (2) for $B$ far from the boundary of $F: \forall$ good sphere $S(p, r)$ such that $N N(p, F)=p^{\prime} \in B, S(p, r) \cap F$ must be contained in the weakly shaded region.

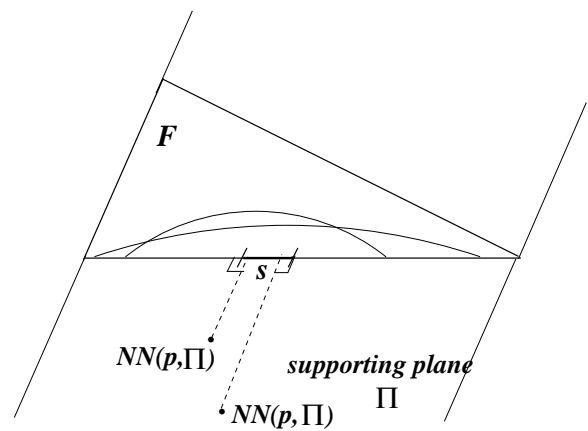

(a)

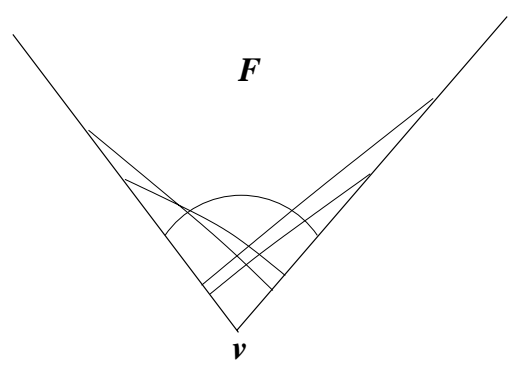

(b)

Figure 3: Illustration of Lemma 4 (3) and (4): Each solid circular arc is the boundary of $S(p, r) \cap F$ for some good sphere $S(p, r)$ such that in (a) $N N(p, F)=$ $p^{\prime} \in s$ and in (b) $N N(p, F)=v$, respectively. Note that the centers of the arcs are $N N(p, \Pi)$ where $\Pi$ is the supporting plane of $F$. The union of all possible regions $S(p, r) \cap F$, i.e., $M_{F}(s)$ in (a) and $M_{F}(v)$ in (b), respectively, has area $\leq c_{F} \frac{\log ^{3} n}{n}$.
This Corollary will be our major tool in proving Theorem 4. To employ it properly we will need to be able to efficiently bound $G(X)$. We will do this using the following:

Lemma 4. Let $F \subset \mathbb{R}^{3}$ be a triangle. Then There exists constant $c_{F}>0$ such that

(1) Let $p^{\prime} \in I(F)$. Then

$$
\operatorname{Area}\left(M_{F}\left(p^{\prime}\right)\right)=\frac{\log ^{2} n}{n} .
$$

(2) Let $B \subset I(F)$ be an $\frac{1}{\sqrt{n}} \times \frac{1}{\sqrt{n}}$ square. Then

$$
\operatorname{Area}\left(M_{F}(B)\right) \leq c_{F} \frac{\log ^{2} n}{n} .
$$

(3) Let $s \subset E(F)$ be an edge segment ${ }^{2}$ with length $(s) \leq$ $\frac{\log n}{\sqrt{n}}$. Then

$$
\operatorname{Area}\left(M_{F}(s)\right) \leq c_{F} \frac{\log ^{3} n}{n}
$$

(4) Let $v \in V(F)$ be a vertex of $F$. Then

$$
\operatorname{Area}\left(M_{F}(v)\right) \leq c_{F} \frac{\log ^{3} n}{n} .
$$

In this extended abstract we do not give the full proof of this lemma. To provide the intuition as to why it is correct suppose that $p^{\prime} \in I(F)$. Let $\Pi$ be the supporting plane of $F$. Then $M_{F}\left(p^{\prime}\right)$ is exactly $C_{\Pi}\left(p^{\prime}, r\right) \cap F$ where $r$ is the unique value such that $\operatorname{Area}\left(C_{\Pi}\left(p^{\prime}, r\right) \cap F\right)=\frac{\log ^{2} n}{n}$. This proves (1). To prove (2) we examine the union of all such discs whose center can be in the small square $B$. See Figure 2. The proofs of (3) and (4) are much more tedious and require a detailed case-by-case analysis. This analysis can be found in the proof of Lemma 3 in [5]. See Figure 3.

As a first consequence of Lemma 4 let $X=S\left(p^{\prime}, r\right)$ be any ball; we will find a general bound on $\left|D_{X}\left(P_{n}\right)\right|$. For all $i$ let $\Pi_{i}$ be the supporting plane of $F_{i}$ and $p_{i}^{\prime \prime}=N N\left(p^{\prime}, \Pi_{i}\right)$. Now let $p \in X$. If $N N\left(p, F_{i}\right) \in I\left(F_{i}\right)$ then $N N\left(p, F_{i}\right) \in$ $C_{\Pi_{i}}\left(p_{i}^{\prime \prime}, r\right)$, i.e., the projection of $X$ on $\Pi_{i}$. See Figure 4 . Such a disk can be covered by $4 n r^{2}$ squares of size $\frac{1}{\sqrt{n}} \times \frac{1}{\sqrt{n}}$. So, from part (2) of the Lemma,

$$
\begin{aligned}
& \text { Area }\left(\bigcup\left\{G_{i}(p): p \in X \text { and } N N\left(p, F_{i}\right) \in I\left(F_{i}\right)\right\}\right) \\
& \quad \leq 4 n r^{2} c_{F_{i}} \frac{\log ^{2} n}{n}=4 c_{F_{i}} r^{2} \log ^{2} n .
\end{aligned}
$$

If $N N\left(p, F_{i}\right) \in E\left(F_{i}\right)$ it is not hard to see that all such nearest neighbors must be contained in the projection of $X$ on the three edges of $F_{i}$ which can be composed of at most three segments, each of length at most $2 r$. These can be partitioned into $\frac{6 r \sqrt{n}}{\log n}$ segments of length $\leq \frac{\log n}{\sqrt{n}}$. From part (3) of the Lemma,

$$
\begin{aligned}
& \text { Area }\left(\bigcup\left\{G_{i}(p): p \in X \text { and } N N\left(p, F_{i}\right) \in E\left(F_{i}\right)\right\}\right) \\
& \quad \leq \frac{6 r \sqrt{n}}{\log n} c_{F_{i}} \frac{\log ^{3} n}{n}=\frac{6 r c_{F_{i}} \log ^{2} n}{\sqrt{n}} .
\end{aligned}
$$

${ }^{2}$ an edge segment can be either closed, open, or half open, half closed. 


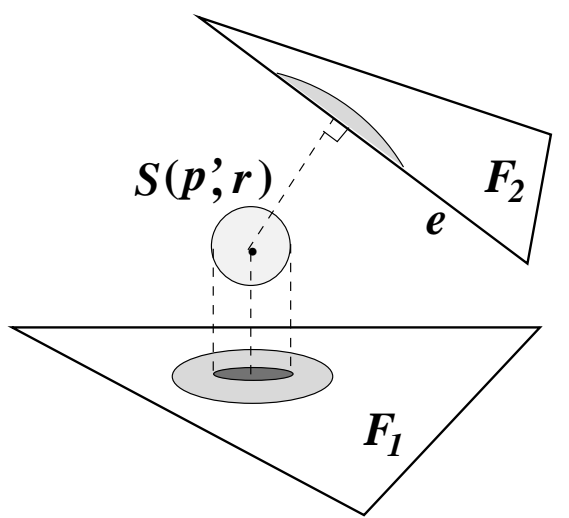

Figure 4: Illustrating $D_{X}\left(P_{n}\right)$ for $X=S\left(p^{\prime}, r\right)$ with $r=\tilde{O}\left(\frac{1}{\sqrt{n}}\right)$. Recall that $D_{X}\left(P_{n}\right)$ is the set of pairs $\left(p_{1}, p_{2}\right) \in P_{n}$ s.t. $p_{1} \in F_{1}, p_{2} \in F_{2}$ and $\left(p_{1}, p_{2}\right)$ are on some $\mathcal{F}$ good sphere $S\left(p^{\prime \prime}, r^{\prime \prime}\right)$ with $p^{\prime \prime} \in X$. The claim is that $p_{1}, p_{2}$ must be in the shaded regions on, respectively, $F_{1}, F_{2}$. The smaller disk in $F_{1}$ is the projection of $S\left(p^{\prime}, r\right)$ onto $F$, more precisely $X_{1}=\cup_{p \in S\left(p^{\prime}, r\right)} N N\left(p, F_{1}\right)$; the bigger disk in $F_{1}$ is $M_{F_{1}}\left(X_{1}\right)$. The shaded region in $F_{2}$ is $M_{F_{2}}\left(X_{2}\right)$ where $X_{2}=\cup_{p \in S\left(p^{\prime}, r\right)} N N(p, F)$.

Finally, from part (4) of the Lemma and the fact that $V\left(F_{i}\right)$ contains only 3 vertices,

$$
\begin{aligned}
& \text { Area }\left(\bigcup\left\{G_{i}(p): p \in X \text { and } N N\left(p, F_{i}\right) \in V\left(F_{i}\right)\right\}\right) \\
& \quad \leq 3 c_{F_{i}} \frac{\log ^{3} n}{n} .
\end{aligned}
$$

Combining the above we have

$$
\operatorname{Area}\left(G_{i}(X)\right)=O\left(r^{2} \log ^{2} n+\frac{r \log ^{2} n}{\sqrt{n}}+\frac{\log ^{3} n}{n}\right) .
$$

In particular, if $r=\tilde{O}\left(\frac{1}{\sqrt{n}}\right)$ then $\operatorname{Area}\left(G_{i}(X)\right)=\tilde{O}\left(\frac{1}{n}\right)$ and $\operatorname{Area}(G(X)) \leq \sum_{i=1}^{2} \operatorname{Area}\left(G_{i}(X)\right)=\tilde{O}\left(\frac{1}{n}\right)$ as well. Applying Corollary 3 , we have just proven:

Lemma 5. If $X=S\left(p^{\prime}, r\right)$ with $r=\tilde{O}\left(\frac{1}{\sqrt{n}}\right)$ then

$$
\operatorname{Pr}\left(\left|D_{X}\left(P_{n}\right)\right|=\tilde{O}(1)\right)=1-n^{-\Omega(\log n)} .
$$

\section{PROOF OF THEOREM 4}

We will now prove Theorem 4 by doing a case-by-case analysis for the 9 different possible labels $L=\left(L_{1}, L_{2}\right) \in\{V, E, I\}^{2}$ and proving (2) for each one. As we will see, after symmetry and other reductions, there will only be three distinct cases.

In what follows let $\Pi_{1}$ and $\Pi_{2}$, respectively, be the supporting planes of $F_{1}$ and $F_{2}$.

Case 1: $L_{1}=V$. (The case $L_{2}=V$ is symmetric.)

This case is very simple and can be proven directly without using the tools developed in the previous section. Suppose

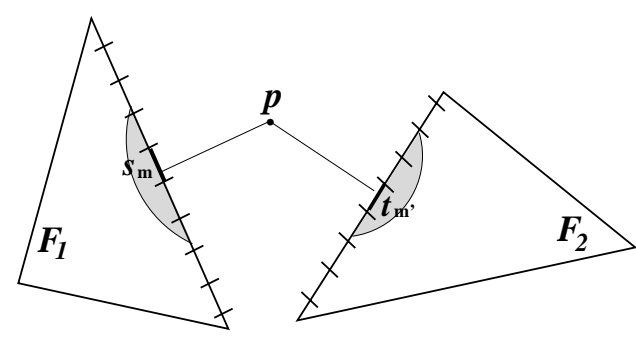

Figure 5: The case $L=(E, E)$. Knowing $N N\left(p, F_{1}\right)$ is on segment $s_{m}$ and $N N\left(p, F_{2}\right)$ is on segment $t_{m^{\prime}}$ restricts the locations of $p_{1}$ and $p_{2}$ to, respectively, the shaded regions $M_{F_{1}}\left(s_{m}\right)$ and $M_{F_{2}}\left(t_{m^{\prime}}\right)$.

$\left(p_{1}, p_{2}\right) \subseteq P_{n} \cap S(p, r)$ for some $\mathcal{F}$-good sphere $S(p, r)$ with $L_{1}(p)=V$ and $L_{2}(p) \in\{V, E, I\}$.

By definition we have that $p_{1} \in M_{F_{1}}\left(V\left(F_{1}\right)\right)$. From Lemma 4 (4), we know that $\operatorname{Area}\left(\cup_{v \in V\left(F_{1}\right)} M_{F_{1}}(v)\right) \leq 3 c_{F_{1}} \frac{\log ^{3} n}{n}$. Plugging into the formula for the Poisson distribution, this gives

$$
\operatorname{Pr}\left(\left|P_{n} \cap M_{F_{1}}\left(V\left(F_{1}\right)\right)\right| \geq 6 c_{F_{1}} \log ^{3} n\right)=n^{-\Omega(\log n)} .
$$

Again directly from the formula for the Possion distribution and simple calculations we get that $\operatorname{Pr}\left(\left|P_{n} \cap F_{2}\right| \geq\right.$ $\left.2 n \operatorname{Area}\left(F_{2}\right)\right)=n^{-\Omega(\log n)}$.

Therefore, with probability $1-n^{-\Omega(\log n)}$, the total number of such $\left(p_{1}, p_{2}\right)$ is $\leq\left(6 c_{F_{1}} \log ^{3} n\right)\left(2 n\right.$ Area $\left.\left(F_{2}\right)\right)=\tilde{O}(n)$ and we are done.

Case 2: $L_{1}=L_{2}=E$.

See Figure 5. Partition the edges of $F_{1}, F_{2}$ into, respectively, $l_{1}=O\left(\frac{\sqrt{n}}{\log n}\right)$ smaller segments $s_{1}, s_{2}, \ldots, s_{l_{1}}$ and $l_{2}=O\left(\frac{\sqrt{n}}{\log n}\right)$ segments $t_{1}, t_{2}, \ldots, t_{l_{2}}$, each of length $\leq \frac{\log n}{\sqrt{n}}$. $\forall m_{1} \leq l_{1}, \forall m_{2} \leq l_{2}$, set

$X_{m_{1}, m_{2}}=\left\{p \in R_{L}: N N\left(p, F_{1}\right) \in s_{m_{1}}, N N\left(p, F_{2}\right) \in t_{m_{2}}\right\}$.

Since $R_{L}=\cup_{1 \leq m_{1} \leq 1_{1}, 1 \leq m_{2} \leq 1_{2}} X_{m_{1}, m_{2}}$ we have

$$
\left|D_{R_{L}}\left(P_{n}\right)\right| \leq \sum_{1 \leq m_{1} \leq 1_{1}, 1 \leq m_{2} \leq 1_{2}}\left|D_{X_{m_{1}, m_{2}}}\left(P_{n}\right)\right| .
$$

By definition $G_{1}\left(X_{m_{1}, m_{2}}\right) \subseteq M_{F_{1}}\left(s_{m_{1}}\right)$ and $G_{2}\left(X_{m_{1}, m_{2}}\right) \subseteq$ $M_{F_{2}}\left(t_{m_{2}}\right)$. By Lemma 4 ,

$\operatorname{Area}\left(G\left(X_{m_{1}, m_{2}}\right)\right) \leq \sum_{i=1}^{2} \operatorname{Area}\left(G_{i}\left(X_{m_{1}, m_{2}}\right)\right)=O\left(\frac{\log ^{3} n}{n}\right)$

so applying Corollary 3 gives

$$
\operatorname{Pr}\left(\left|D_{X_{m_{1}, m_{2}}}\left(P_{n}\right)\right|=\tilde{O}(1)\right)=1-n^{-\Omega(\log n)} .
$$

Summing over all $m_{1}, m_{2}$ yields

$$
\operatorname{Pr}\left(\left|D_{R_{L}}\left(P_{n}\right)\right|=\tilde{O}(n)\right)=1-n^{-\Omega(\log n)} .
$$




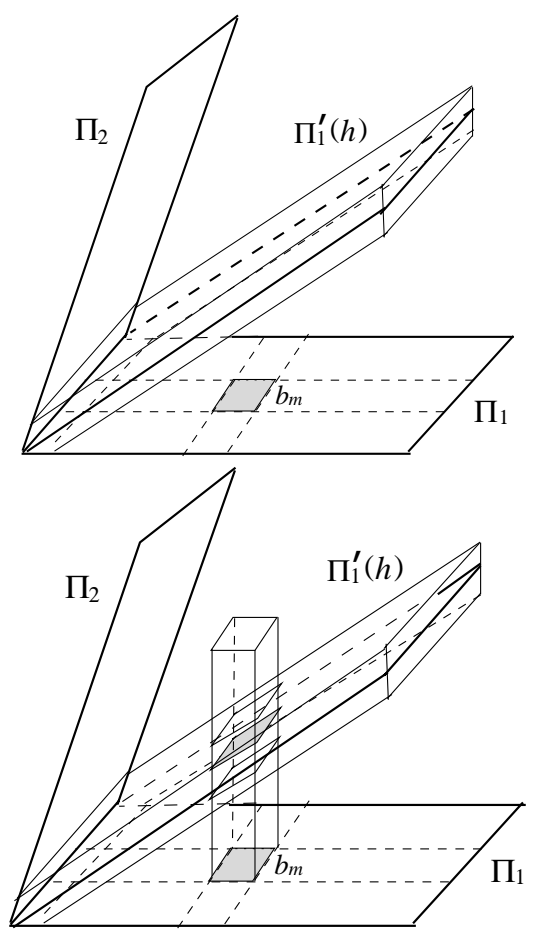

Figure 6: In the top figure we see half of the plane $\Pi_{1}^{\prime}$ which is one of the bisecting planes of $\Pi_{1}$ and $\Pi_{2}$. $\Pi_{1}^{\prime}(h)$ is the sandwich of width $2 h$ around $\Pi_{1}^{\prime}$, In the bottom figure we take a square $b_{m}$ on $\Pi_{1}$ and look at $X_{m}^{1}$, the intersection of the infinite square prism through $b_{m}$ perpendicular to $\Pi_{1}$ and the sandwich. If $N N\left(p, F_{1}\right) \in b_{m}$ and there is some $r$ such that $S(p, r)$ is good sphere that intersects both $F_{1}$ and $F_{2}$, then $p \in X_{m}^{1}$.

Case 3(a): $L_{1}=L_{2}=I$ and $F_{1} \not \mid F_{2}$.

See Figure 6. Recall that $\Pi_{1}, \Pi_{2}$ are the supporting planes of $F_{1}$ and $F_{2}$. Define $\Pi_{1}^{\prime}$ and $\Pi_{2}^{\prime}$ to be the bisecting planes of $\Pi_{1}, \Pi_{2}$, i.e., the two planes that contain the line $\Pi_{1} \cap \Pi_{2}$ and satisfy

$$
\Pi_{1}^{\prime} \cup \Pi_{2}^{\prime}=\left\{p: d\left(p, \Pi_{1}\right)=d\left(p, \Pi_{2}\right)\right\} .
$$

( $\Pi_{1}^{\prime}, \Pi_{2}^{\prime}$ exist because $\Pi_{1}$ and $\Pi_{2}$ are not parallel). For arbitrary plane $\Pi$ define the $h$-sandwich around $\Pi$ as

$$
\Pi(h)=\{p: d(p, \Pi) \leq h\} .
$$

Straightforward geometric arguments (omitted in this extended abstract) show that

$$
\text { if } \begin{aligned}
p \notin \Pi_{1}^{\prime}\left(\frac{\log ^{2} n}{\sqrt{n}}\right) \cup \Pi_{2}^{\prime}\left(\frac{\log ^{2} n}{\sqrt{n}}\right), \\
\quad \text { then }\left|d\left(p, \Pi_{1}\right)-d\left(p, \Pi_{2}\right)\right|=\Omega\left(\frac{\log ^{2} n}{\sqrt{n}}\right) .
\end{aligned}
$$

Now suppose that $p$ is such that $L_{1}(p)=L_{2}(p)=I$ and $p \notin \Pi_{1}^{\prime}\left(\frac{\log ^{2} n}{\sqrt{n}}\right) \cup \Pi_{2}^{\prime}\left(\frac{\log ^{2} n}{\sqrt{n}}\right)$. If $S(p, r)$ is any sphere that intersects both $F_{1}$ and $F_{2}$, then

$$
r \geq \max \left(d\left(p, \Pi_{1}\right), d\left(p, \Pi_{2}\right)\right) .
$$

This would imply that $S(p, r)$ would have to contain at least one of the two closed disks $C_{\Pi_{1}}\left(N N\left(p, F_{1}\right), r^{\prime}\right)$

or $C_{\Pi_{2}}\left(N N\left(p, F_{2}\right), r^{\prime}\right)$ for some $r^{\prime}=\Omega\left(\frac{\log ^{2} n}{\sqrt{n}}\right)$. By definition, $S(p, r)$ can therefore not be both $F_{1}$-good and $F_{2}$-good and can therefore not be $\mathcal{F}$-good.

We have therefore just seen that if $S(p, r)$ is a $\mathcal{F}$-good sphere then $p \in \Pi_{1}^{\prime}\left(\frac{\log ^{2} n}{\sqrt{n}}\right) \cup \Pi_{2}^{\prime}\left(\frac{\log ^{2} n}{\sqrt{n}}\right)$. In particular, this implies

$$
R_{L} \in \Pi_{1}^{\prime}\left(\frac{\log ^{2} n}{\sqrt{n}}\right) \cup \Pi_{2}^{\prime}\left(\frac{\log ^{2} n}{\sqrt{n}}\right) .
$$

We now cover the interior of $F_{1}$ by $l=O(n)$ squares $b_{1}, b_{2}, \ldots, b_{l}$, each of size $\frac{1}{\sqrt{n}} \times \frac{1}{\sqrt{n}} \cdot \forall m \leq l$, set

$$
X_{m}=\left\{p \in R_{L}: N N\left(p, F_{1}\right) \in b_{m}\right\} .
$$

By definition, $R_{L}=\cup_{m=1}^{l} X_{m}$, so

$$
\left|D_{R_{L}}\left(P_{n}\right)\right| \leq \sum_{m=1}^{l}\left|D_{X_{m}}\left(P_{n}\right)\right| .
$$

From the argument above we know that $X_{m} \subset \Pi_{1}^{\prime}\left(\frac{\log ^{2} n}{\sqrt{n}}\right) \cup$ $\Pi_{2}^{\prime}\left(\frac{\log ^{2} n}{\sqrt{n}}\right)$. For $j=1,2$, set $X_{m}^{j}=X_{m} \cap \Pi_{j}^{\prime}\left(\frac{\log ^{2} n}{\sqrt{n}}\right)$. Each $X_{m}^{j}$ is contained in the intersection of an infinite square prism with $\frac{1}{\sqrt{n}} \times \frac{1}{\sqrt{n}}$ crossection and a sandwich of width $\frac{\log ^{2} n}{\sqrt{n}}$ so there exists $p_{m}^{j}, r_{m}^{j}$ with $r_{m}^{j}=O\left(\frac{\log ^{2} n}{\sqrt{n}}\right)$ such that $X_{m}^{j} \subset S\left(p_{m}^{j}, r_{m}^{j}\right)$. Applying Lemma 5 shows that

$$
\operatorname{Pr}\left(\left|D_{X_{m}^{j}}\left(P_{n}\right)\right|=\tilde{O}(1)\right)=1-n^{-\Omega(\log n)} .
$$

Since $\left|D_{R_{L}}\left(P_{n}\right)\right| \leq \sum_{m, j}\left|D_{X_{m}^{j}}\left(P_{n}\right)\right|$, summing over all $m, j$ yields

$$
\operatorname{Pr}\left(\left|D_{R_{L}}\left(P_{n}\right)\right|=\tilde{O}(n)\right)=1-n^{-\Omega(\log n)} .
$$

Case 3(b): $L_{i}=L_{j}=I$ and $F_{i} \| F_{j}$.

As before, let $\Pi_{1}, \Pi_{2}$ be the supporting planes of $F_{1}, F_{2}$. Now, let $\Pi_{3}$ be the plane parallel to $\Pi_{1}, \Pi_{2}$ that is equidistant from both. If $p$ has $L_{1}(p)=L_{2}(p)=I$ then a straightforward geometric argument similar to the one in Case 3 (a) shows that if, for some $r \geq 0, S(p, r)$ is $\mathcal{F}$-good, then $p \in \Pi_{3}\left(\frac{\log ^{2} n}{\sqrt{n}}\right)$.

We now repeat the same procedure. Ccover the interior of $F_{1}$ by $l=O(n)$ squares $b_{1}, b_{2}, \ldots, b_{l}$ each of size $\frac{1}{\sqrt{n}} \times \frac{1}{\sqrt{n}}$. $\forall m \leq l$, set $X_{m}=\left\{p \in R_{L}: N N\left(p, F_{1}\right) \in b_{m}\right\}$. Since $R_{L}=\cup_{m=1}^{l} X_{m}$, we have

$$
\left|D_{R_{L}}\left(P_{n}\right)\right| \leq \sum_{m=1}^{l}\left|D_{X_{m}}\left(P_{n}\right)\right| .
$$

Note that, from our discussion above, $X_{m} \in \Pi_{3}\left(\frac{\log ^{2} n}{\sqrt{n}}\right)$ so $X_{m}$ is contained in the intersection of an infinite rectangular prism with $\frac{1}{\sqrt{n}} \times \frac{1}{\sqrt{n}}$ crossection and a sandwich 


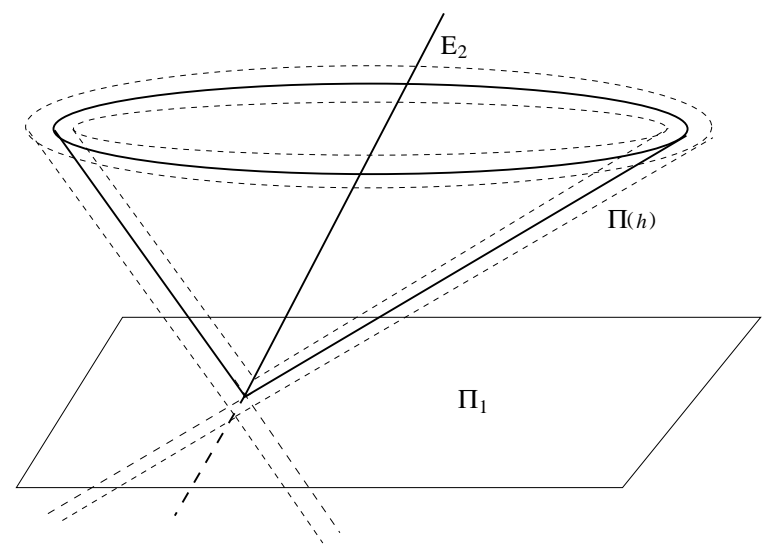

Figure 7: The cone that is the bisecting surface of $\Pi_{1}$ and $E_{2}$ and its $h$-sandwich.

of width $\frac{\log ^{2} n}{\sqrt{n}}$. It therefore must be contained in some ball of radius $O\left(\frac{\log ^{2} n}{\sqrt{n}}\right)$. Applying Lemma 5 shows that $\operatorname{Pr}\left(\left|D_{X_{m}}\left(P_{n}\right)\right|=\tilde{O}(1)\right)=1-n^{-\Omega(\log n)}$. Summing over all $m$ yields

$$
\operatorname{Pr}\left(\left|D_{R_{L}}\left(P_{n}\right)\right|=\tilde{O}(n)\right)=1-n^{-\Omega(\log n)} .
$$

Case 4: $L_{1}=I$ and $L_{2}=E$. (The case $L_{1}=E$ and $L_{2}=I$ is symmetric)

See Figure 7. Fix $e$ to be one of the three edges of $F_{2}$ and assume that $N N\left(p, F_{2}\right) \in e$.

We will prove the theorem for each fixed $e$ separately; summing over the three edges will prove it for the full case

Let $E_{2}$ be the full line of which $e$ is a segment. Define the bisecting surface (Figure 7 ) of $\Pi_{1}$ and $E_{2}$ to be

$$
\Pi:=\left\{p: d\left(p, \Pi_{1}\right)=d\left(p, E_{2}\right)\right\} .
$$

If $\Pi_{1} \| E_{2}$, then $\Pi$ is a paraboloid; otherwise $\left(\Pi_{1} \nvdash E_{2}\right), \Pi$ is an infinite double cone passing through the intersection point $\Pi_{1} \cap E_{2}$. Define the $h$-sandwich around $\Pi$ to be

$$
\Pi(h)=\{p: d(p, \Pi) \leq h\} .
$$

Using straightforward geometric arguments (omitted in this extended abstract) we can show that $\forall p$ such that $L_{1}(p)=$ $I, L_{2}(p)=E$, and $N N\left(p, F_{2}\right) \in e$, if $p \notin \Pi\left(\frac{\log n}{\sqrt{n}}\right)$ then $\left|d\left(p, \Pi_{1}\right)-d\left(p, E_{2}\right)\right|=\Omega\left(\frac{\log n}{\sqrt{n}}\right)$. So $\forall p$ such that $L_{1}(p)=$ $I, L_{2}(p)=E$ and $N N\left(p, F_{2}\right) \in e$, if $p \notin \Pi\left(\frac{\log n}{\sqrt{n}}\right)$ and $S(p, r)$ is any sphere that intersects both $F_{1}$ and $e$, then $r \geq \max \left(d\left(p, \Pi_{1}\right), d\left(p, E_{2}\right)\right)$. This would imply that $S(p, r)$ would have to contain at least one of $C_{\Pi_{1}}\left(N N\left(p, F_{1}\right), r^{\prime}\right)$ or $C_{\Pi_{2}}\left(N N\left(p, E_{2}\right), r^{\prime}\right)$ for some $r^{\prime}=\Omega\left(\frac{\log n}{\sqrt{n}}\right) \cdot S(p, r)$ can therefore not be both $F_{1}$-good and $F_{2}$-good and can therefore not be $\mathcal{F}$-good.
We have just seen that if $S(p, r)$ is a $\mathcal{F}$-good sphere, then $p \in \Pi\left(\frac{\log n}{\sqrt{n}}\right)$. In particular, this implies $R_{L} \in \Pi\left(\frac{\log n}{\sqrt{n}}\right)$.

As in Case 2, we now cover the interior of $F_{1}$ by $l=O(n)$ squares $b_{1}, b_{2}, \ldots, b_{l}$, each of size $\frac{1}{\sqrt{n}} \times \frac{1}{\sqrt{n}} \cdot \forall m \leq l$, set

$$
X_{m}=\left\{p \in R_{L}: N N\left(p, F_{1}\right) \in b_{m}\right\} .
$$

By definition, $R_{L}=\cup_{m=1}^{l} X_{m}$ so $\left|D_{R_{L}}\left(P_{n}\right)\right| \leq \sum_{m=1}^{l}\left|D_{X_{m}}\left(P_{n}\right)\right|$.

From the argument above we know that $X_{m} \subset \Pi\left(\frac{\log n}{\sqrt{n}}\right)$. More precisely, each $X_{m}$ is contained in the intersection of an infinite square prism with $\frac{1}{\sqrt{n}} \times \frac{1}{\sqrt{n}}$ cross-section $b_{m}$ and a sandwich of width $\frac{\log n}{\sqrt{n}}$. So there exists $p_{m}, r_{m}$ with $r_{m}=$ $O\left(\frac{\log n}{\sqrt{n}}\right)$ such that $X_{m} \subset S\left(p_{m}, r_{m}\right)$. Applying Lemma 5 shows that $\operatorname{Pr}\left(\left|D_{X_{m}}\left(P_{n}\right)\right|=\tilde{O}(1)\right)=1-n^{-\Omega(\log n)}$. Since $\left|D_{R_{L}}\left(P_{n}\right)\right| \leq \sum_{m}\left|D_{X_{m}}\left(P_{n}\right)\right|$, summing over all $m$ yields

$$
\operatorname{Pr}\left(\left|D_{R_{L}}\left(P_{n}\right)\right|=\tilde{O}(n)\right)=1-n^{-\Omega(\log n)} .
$$

\section{ACKNOWLEDGEMENTS}

The work of both authors was partially supported by HK RGC CERG grants 6082/97E and 6137/98E.

Also the authors would like to thank Siu-Wing Cheng for useful discussions about this work.

\section{REFERENCES}

[1] D. Attali and J-D. Boissonnat, "Complexity of the Delaunay Triangulation of Points on Polyhedral Surfaces", INRIA Technical report 4232, (July 2001).

[2] D. Attali and J-D. Boissonnat, "A Linear Bound on the complexity ofthe Delaunay triangulation of points on polyhedral surfaces", Submitted to Solid Modeling 2002. Also available at http://www.inria.fr/sophia/prisme/personnel /boissonnat/Articles/linearDT.ps.gz.

[3] Jeff Erikson, "Nice Point Sets Can have Nasty Delaunay Triangulations", Proceedings of the 17th Annual ACM Symposium on Computational Geometry, (June 3-5, 2001) 96-105.

[4] M. J. Golin and Hyeon-Suk Na, "On the Average Complexity of 3D-Voronoi Diagrams of Random Points On Convex Polytopes", Proceedings of the 12th Canadian Conference on Computational Geometry (CCCG00), (2000) 127-135. Extended version accepted in Computational Geometry: Theory and Applications.

[5] M. J. Golin and Hyeon-Suk Na, "On the Proofs of Two Lemmas Describing the Intersections of Spheres with the Boundary of a Convex Polytope", Hong Kong UST Theoretical Computer Science Center Technical Report HKUST-TCSC-2001-09, July 2001. Available from www.cs.ust.hk/tcsc/RR.

[6] Franco P. Preparata and Michael Ian Shamos, Computational Geometry: An Introduction, Springer-Verlag, New York.(1985). 